\title{
The Design of Bearing Fault Diagnosis Classifier Based on the Bayesian Classification
}

\author{
Chunhe Shi", a, Xiaowei Han ${ }^{1, b}$, and Zhen $\mathrm{Li}^{2, \mathrm{c}}$ \\ ${ }^{1}$ School of Information Engineering, Shenyang University, Shenyang 110044, China \\ ${ }^{2}$ Northeast Regional Air Traffic Management Bureau of CAAC, Shenyang 110043, China \\ aschsydx@163.com, bhxw69@163.com, czlalbert@126.com
}

Keywords: PCA, Bayesian, Fault Detection

\begin{abstract}
The advantages of Principal Component Analysis (PCA) in dimension reduction are obvious, while the classifying methods are various. PCA, applied to Bayesian classification with the minimium risk, is adopted to realize classifying and testing the learning samples in this paper, eventually accurately realizing the classifier training towards the new input sample and then detecting the testing effect.
\end{abstract}

\section{Introduction}

In recent years, the Bayesian has become a primary tool of data mining and knowledge discovery, and achieved good applying effect in classification, clustering, forecasting, and derivation of the rules, etc. Therefore, this paper uses Bayesian classifier to achieve the rolling bearing fault diagnosis. PCA (Principal Component Analysis) is a technique of converting the data set to minor dimensions and components containing all or major information of the original data set via a reversible linear transformation. By using PCA, the complex data will be simplified, thence, it is widely used in data mining, pattern recognition, signal evaluation, signal detection, image coding, and other fields. PCA's principles are as follow:

Let $\mathrm{x}$ be a random vector representing $\mathrm{m}$-dimension. Assuming that the $\mathrm{x}$ mean is zero, i.e.:

$$
E(x)=0
$$

Let $\mathrm{w}$ be $\mathrm{m}$-dimensional unit vector, and $\mathrm{x}$ the mapped in $\omega$. The mapping is defined as the inner product of the vector $\mathrm{x}$ and $\omega$, expressed as:

$$
y=\sum_{k=1}^{n} W_{k} X_{K}=W^{T} X
$$

Satisfying the constraints

$$
\|w\|=\left(w^{T} w\right)^{1 / 2}=1
$$

The purpose of PCA is searching for a weight vector $w$, which maximizes the value of the expression:

$$
\begin{aligned}
& E\left[y^{2}\right]=E\left[\left(w^{T} x\right)^{2}\right]=w^{T} E\left[x x^{T}\right] w=w^{T} C_{x} w \\
& C_{x} W_{j}=\lambda_{j} w_{j} \quad j=1,2 \because m,
\end{aligned}
$$


i.e., w which maximizes the value of equation (4) is the corresponding eigenvector to the largest eigenvalue of the matrix.

Considering the advantages of PCA, it is herein introduced to reduce and demensions of data sets and process the data, in order to improve the efficiency of Bayesian classifier and simplify the structure.

\section{Basic Principles of PCA and Bayesian Classification}

\subsection{Basic Principles of PCA}

PCA is a statistical analysis method on which multiple features are mapped as a few comprehensive characteristics. Adopting dimension reduction, PCA finds several variables to represent the multiple original features, making these variables reflect the original information to the utmost and irrelavant to each other, so as to achieve the objective of simplification.

Its basic idea is to attempt to reassemble the multiple related indexes $\mathrm{X}_{1}, \mathrm{X}_{2}, \ldots, \mathrm{X}_{\mathrm{P}}$ (for example $\mathrm{p}$ indexs) into a reduced number of disparate set of comprehensive index $\mathrm{F}_{\mathrm{m}}$ to replace the original ones. How to extract Indexes, so that it can both reflect to the maximum the information represented by the variable $X_{p}$, and ensure new indexes' irrelavance (without information overlapping).

Suppose that $F_{1}$ represents the principal component index formed by the first linear combination of the original variable, i.e. $F_{1}=a_{11} X_{1}+a_{21} X_{2}+\ldots+a_{p 1} X_{p}$, the amount of information extracted by each principal component can be measured via its variance. The higher the variance $\operatorname{Var}\left(\mathrm{F}_{1}\right)$ is, the more information $F_{1}$ contains. Usually, the first principal component $F_{1}$ is suppoed to be the most informative, therefore, the variance of $F_{1}$, selected from all the linear combinations, ought to be the largest among $\mathrm{X}_{1}, \mathrm{X}_{2}, \ldots, \mathrm{X}_{\mathrm{P}}$, thus $\mathrm{F}_{1}$ is named the first principal component. If the first principal component is not able to represent the original information of $\mathrm{p}$ indexes, consider selecting a second principal component index $\mathrm{F}_{2}$. To effectively reflect the original information, the information contained in $F_{1}$ does not necessarily appear in $F_{2}$, i.e., $F_{2}$ and $F_{1}$ keep independent and irrelavant. Expressed in mathematics language, its covariance $\operatorname{Cov}\left(F_{1}, F_{2}\right)=0$, so $F_{2}$ is of the maximium variance among all the linear combination, say, $\mathrm{X}_{1}, \mathrm{X}_{2}, \ldots, \mathrm{X}_{\mathrm{P}}$, thus $\mathrm{F}_{2}$ is named the second principal component, and by analogy $F_{1}, F_{2}, \ldots, F_{m}$ are the first, second, ..., $m$-th principal components of the original variables $\mathrm{X}_{1}, \mathrm{X}_{2}, \ldots \mathrm{X}_{\mathrm{P}}$.

According to the above analysis,

(1) the $F_{i}$ and $F_{j}$ are irrelavant, namely $\operatorname{Cov}\left(F_{i}, F_{j}\right)=0$.

(2) $F_{1}$ is of the maximium variance among all the linear combinations (the coefficients satisfy the above requirements), say, $F_{1}$ are $X_{1}, X_{2}, \ldots, X_{P}$; i.e., $F m$ is of the maximium variance among all the linear combinations $X_{1}, X_{2}, \ldots, X_{P}$ irrelavant to $F_{1}, F_{2}, \ldots \ldots, F_{m-1} . F_{1}, F_{2}, \ldots, F_{m}(m \leq p)$ are the new constructed variables, i.e. the first, second, ... ..., $\mathrm{m}$-th principal components of the original variables.

\subsection{Basic Principles of Bayesian Classification}

Due to the complexity of objective things, the risks of classifier judgement are not identical. The risk of misjudging cancer cells as normal ones, for example, is greater than that of miscalculating normal cells as cancer ones. Therefore, the concept of risk is introduced in Bayesian classifiers. In practical application, the magnitude of risks is identified according to specific situations, usually represented by a set of coefficients $C_{\mathrm{ij}}$. $\mathrm{C}_{\mathrm{ij}}$ refers to the risk when classifier is classified as $\omega_{\mathrm{i}}$ by the recognition samples, while the actual classificaton of the sample is $\omega_{\mathrm{j}}$. The basic idea of designing a classifier with minimal risk is to calculate the conditional risk of classifying $\mathrm{x}$ into $\omega_{\mathrm{i}}$ via the posterior probability calculation. 
Comparing each value of $\mathrm{R}_{\mathrm{i}}(\mathrm{x})$, the classification corresponding to the minimum is the result. Since the standard of evaluating classifiers is average risk, this classifier is of the minimum average risk. In practical application, posterior probabilities are difficult to obtain. According to the number of pattern classificatoin and value-reading pattern of $\mathrm{C}_{\mathrm{ij}}$ categories, a variety of classifiers are able to be designed, for example, when patterns contain two classifications, discriminant functions are

$$
\begin{aligned}
& g_{1}(x)=\left(C_{21}-C_{11}\right) P\left(x \mid w_{1}\right) P\left(w_{1}\right) \\
& g_{2}(x)=\left(C_{12}-C_{22}\right) P\left(x \mid w_{2}\right) P\left(w_{2}\right)
\end{aligned}
$$

Select $\mathrm{C}_{11}, \mathrm{C}_{22}$ as zero, $\mathrm{C}_{12}$ and $\mathrm{C}_{21}$ as 1 , and this brings about a classifier of two classifications with minimum error probability. Actually, the minimum error probability classifier is a special case of minimal risk classifiers.

\section{Experimental Procedure}

(1) Selecting all samples of different classifications to form Matrix $X_{n \times N}$, with the testing sample is $\mathrm{X}_{\mathrm{n} \times 1}$.

(2) Calculating the covariance matrix $S_{n \times n}$ of $X_{n \times N}$.

(3) Calculating the eigenvalue $\lambda_{1} \geq \lambda_{2} \geq \cdots \geq \lambda_{n}$ of $S_{n \times n}$.

(4) According to a certain contribution rate, selecting the first m columns of $\mathrm{C}_{\mathrm{n} \times \mathrm{n}}$, constituting $\mathrm{C}_{\mathrm{n} \times \mathrm{m}}$.

(5) Calculating the sample component $X_{m \times n}=C_{n \times m} X_{n \times N}$ from sample gallery and the principal component $\mathrm{X}_{\mathrm{m} \times 1}=\mathrm{CT}_{\mathrm{n} \times \mathrm{m}} \mathrm{X}_{\mathrm{n} \times 1}$.

(6) Calculating the mean value of each classification of digital samples

$$
\overline{X^{\left(w_{i}\right)}}=\frac{1}{N_{i}} \sum_{X \in w_{i}} X=\left(\overline{x_{1}^{\left(w_{i}\right)}}, \overline{x_{2}^{\left(w_{i}\right)}}, \cdots, \overline{x_{n}^{\left(w_{i}\right)}}\right)^{T} \quad i=0,
$$

In the above equation, $\mathrm{Ni}$ represents the number of samples of the $\mathrm{w}_{\mathrm{i}}$ classification and $\mathrm{n}$ represents the number of features.

(7) Evaluating covariance matrix of each classification.

$$
S_{j k}^{i}=\frac{1}{N_{i}-1} \sum_{l=1}^{N_{i}}\left(x_{l j}-\overline{x_{j}^{\left(w_{i}\right)}}\right)\left(x_{l k}-\overline{x_{k}^{\left(w_{i}\right)}}\right) \quad j, k=1,2, \quad,
$$

In the above equation, $l$ stands for the serial number of samples in the $\mathrm{w}_{\mathrm{i}}$ classification, in which $l=0,1,2, \cdots, N_{i}$

$x_{l j}$ represents the $\mathrm{j}$-th feature value of the l-th sample in $\mathrm{w}_{\mathrm{i}}$ classification;

$\overline{x_{j}^{\left(w_{i}\right)}}$ represents the average value of the $\mathrm{j}$-th feature of a $\mathrm{N}_{\mathrm{i}}$ number of samples in $\mathrm{w}_{\mathrm{i}}$ classification;

$x_{l k}$ represents the $\mathrm{k}$-th feature value of the 1-th sample in $\mathrm{w}_{\mathrm{i}}$ classification;

$\overline{x_{k}^{\left(w_{i}\right)}}$ represents the average value of the $\mathrm{k}$-th feature of a $\mathrm{N}_{\mathrm{i}}$ number of samples in $\mathrm{w}_{\mathrm{i}}$ classification;

$x_{l j}$ represents the $\mathrm{j}$-th feature value of the 1 -th sample in $\mathrm{w}_{\mathrm{i}}$ classification; 
(8) Calculating the inverse matrix $S_{i}^{-1}$ of each classification's covariance matrix, and the determinant $\left|S_{i}\right|$ of the covariance matrix.

(9) Evaluating the prior probability of each classification.

$P\left(w_{i}\right) \approx N_{i} / N \quad i=0,1$

In the above equation, $\mathrm{P}\left(\mathrm{w}_{\mathrm{i}}\right)$ is the prior probability of number classification; $\mathrm{N}_{\mathrm{i}}$ is the number of samples of number $\mathrm{i}$; and $\mathrm{N}$ is the total number of samples.

(10) Calculating the posterior probability $P[i], \quad i=0,1$.

$$
P[i]=-\frac{1}{2}\left(X-\overline{X^{\left(w_{i}\right)}}\right)^{T} S_{i}^{-1}\left(X-\overline{X^{\left(w_{i}\right)}}\right)+\ln P\left(w_{i}\right)-\frac{1}{2} \ln \left|S_{i}\right|
$$

(11) Defining the array of loss as loss[2][2], and setting the initial value as

$\operatorname{loss}[i][j]=\left\{\begin{array}{l}0, i=j \\ 1, i \neq j\end{array}\right.$

(12) Calculating the loss of each classification

$\operatorname{risk}[i]=\sum_{j=0}^{1} \operatorname{loss}[i][j] p[j]$

(13) Finding the classification corresponding to the minimum loss, accordingly identifying this classification as the one that testing samples belong to.

\section{Experimental Results and Analysis}

Using PCA after the dimension structure of the Bayesian classification and unused dimension data learning results on classification accuracy differ in structural classification accuracy is much higher than other classifiers, also using the dimension data have nodes small, simple structure and high efficiency. Use the test sample test result, but through this classifier classification classification is fully consistent with the test sample, this classifier is very accurate.

\section{Conclusion}

Bayesian classifier of low efficiency of shortcomings, presented data set through the PCA principal components analysis dimensionality reduction. Reduce data, then data dimension reduction method for Bayesian learning, improved operational efficiency and by increasing the efficiency of correctness to ensure better results. In addition, this method is simple, high recognition rate, in real time, trained networks have very good classification abilities.

\section{References}

[1]. Yang Shuying. Pattern recognition and intelligent-Matlab technologies. Electronic Industry Press, 2008, p. 132-140.

[2]. Zeng Huanglin, Yu Jue-bang, Zeng Qian. Feature simplification based on principal components analysis. Journal of Sichuan Institute of light industry, Vol. 12(1999) No. 1, p. 1-4.

[3]. N.Huang. Based on PCA Bayesian network classifiers. Mathematical statistics and management, Vol. 18(1999) No.5, p. 44-52.

[4]. Chen Y D, Du R. Fault features of large rotating machinery and diagnosiusing sensor fusion. Journal of Sound and Vibration, Vol. 188(1995) No.2, p. 227-242. 